\title{
Detection of Micrometasteses In Lymph Nodes in Cases of Carcinoma Breast by Immunohistochemistry
}

\author{
Sunita Sharma ${ }^{1 *}$ and CS Mohanty ${ }^{2}$ \\ ${ }^{1}$ Dept of Pathology INHS Asvini, Mumbai, India \\ ${ }^{2}$ Dept of Undersea medicine, INHS Asvini, Mumbai, India
}

\begin{abstract}
Background: We evaluated archived lymph nodes of breast carcinoma for micrometastases detection by serial multiple sections (SMS) and immunohistochemistry (IHC) and found increased positive lymph nodes yield which led to change in staging in few cases, treatment and prognosis.

Method: Metastatic free, early breast cancer cases of 36 patients with lymph node status pN0 or pN1 were evaluated. These were the cases had been treated with modified radicle mastectomy and axillary lymph node dissection. All the lymph node was cut at $50 \mu \mathrm{m}$ interval to get $5 \mu \mathrm{m}$ thick serial sections. These serial sections (SMS) were stained with Hematoxyline and Eosin (H\&E). Out of these 2 sections were subjected to Immunohistochemistry (IHC). Monoclonal antibodies chosen for IHC were panCK (Cytokeratin) and EMA (Epithelial membrane antigen) to detect micrometasteses.
\end{abstract}

Results: Total 463 lymph nodes from 36 cases of carcinoma breast were studied. New serial sections (SMS) cut from 435 lymph nodes of breast revealed 16(3.67\%) additional positive lymph nodes from 12 cases. Out of 16 positive lymph nodes 4 were macrometasteses and 12 were micrometastases. Immunostaining with CK antibody revealed micrometasteses in 40 of 435 (9.19\%) lymph nodes and by EMA antibody 33 of $435(7.59 \%)$. Out of 40 micrometasteses 8 were positive for isolated tumor cells (ITC). We calculated the $Z$ value and corresponding $\mathrm{p}$ value between the two methods as H\&E versus SMS, H\&E versus IHC and SMS versus IHC forcarcinoma breasts. The tests revealed that SMS and IHC are definitely the superior methods in detection of a greater number of positive lymph nodesin cases of carcinoma breast.

$\mathrm{P}$ value for detection of micrometastesesH\&E versus SMS is $<0.05$ (S), H\&E versus $\mathrm{IHC}<0.01$ (S) and SMS versus IHC ( $<0.02$ ). Out of 36 cases 06 cases showed change in staging and 03 out of 06 cases were upstaged from early to advanced stage carcinoma.

Conclusion: Significant number of metasteses are missed by routine processing of lymph nodes on H\&E staining. SMS and IHC increase the yield of metasteses in lymph nodes. CK is superior marker than EMA.

\section{Keywords: Micrometasteses, Immunohistochemistry, Serial Multiple Sections, Isolated Tumor Cells, Cytokeratin, Epithelial Membrane Antigen.}

\section{Introduction}

On global basis, carcinoma breast is the commonest carcinoma among females. ${ }^{[1,5]}$ The common age is between 35-50 years, but it occurs a decade earlier in India. Mean age is 42 years in India as compared to white women. Lymph node histopathological assessment in cancer is an important aspect of surgical management of breast cancers.

Significance of lymph node metasteses is that the behavior and prognosis of cancers are generally stage dependent. The staging of tumor requires proper assessment of draining lymph nodal status in addition to the primary lesion and the presence or absence of distant metasteses. ${ }^{[2,3]}$ The malignant cells disseminating from primary tumor first harbors in regional draining lymph nodes ie axillary nodes in cases of carcinoma breast. The metasteses has a major prognostic implication and hence evaluation of these is essential for staging of carcinoma and subsequent therapy. Currently the axillary lymph nodes dissection and standard histological examination is inadequate and yields many false negative nodes. Even when there is no lymph node metastases, $20-30 \%$ of the node negative patients show recurrence within 10 years. Hence there is a need to identify patients with node negative breast cancer whom Adjuvant chemotherapy may be useful. ${ }^{[3]}$ These are the high-risk group patients who could be benefited by adjuvant chemotherapy. The evidences support that more accurate staging identify patients of high risk who can gain survival advantage with either the extended surgical procedure itself (complete axillary dissection) or the accompanying adjuvant therapy. On the contrary, the patients which are truly node negative need not be exposed to the complications of more extensive surgical procedure or the toxicities of adjuvant therapy. ${ }^{[8]}$

Traditionally, the nodes from a resected specimen are identified by manual palpation, thereby subjecting the 
identification of these nodes to the pathologist's skill and patience. Generally nodes are bisected and examined by H\&E staining of single section. Using this method of manual palpation to identify the lymph node, followed by light microscopy of a section of that node, skillful pathologists can detect deposits of metastatic disease as small as $100 \mu \mathrm{m}$ in diameter, or approximately the size of 100 cancer cells clustered together. ${ }^{[6]}$

Increased pathologic sampling by serial sectioning of nodes within a pathologic specimen increases the detection of metastatic disease. The magnitude of metastases missed due to tissue block sampling is likely to be larger than $0.1 \mathrm{~mm}$ and is directly dependent on the interval between sections evaluated and the tissue left in the paraffin block after sections are removed, thereby potentially introducing significant sampling error. ${ }^{[6]}$ Serial sectioning of axillary nodes of breast cancer patients increased number of higher staged tumors by detecting more positive nodes.

Micrometasteses for $\mathrm{pN}$ classification, the criterion is size of the metasteses and not the size of lymph node. Metastatic deposits ${ }^{[5,7,8]}$ in lymph nodes are recognized in the following three categories
a. Isolated tumor cells $-<0.2 \mathrm{~mm}$
b. Micrometastasis $-0.2-2.0 \mathrm{~mm}$
c. Replacement metastasis $->2 \mathrm{~mm}$

Isolated tumor cells are included in pN0 category while micrometasteses are counted in $\mathrm{pN} 1$ nodal staging in cases of carcinoma breast. Immunohistochemistry (IHC) of lymph nodes, the technologic advances in the diagnosis allow the detection of micrometasteses in histological negative lymph nodes. Serial multiple sections, though labour intensive and IHC can accurately identify high risk patients by detecting micrometasteses which are negative by conventional method of single H\&E section. ${ }^{[3]}$ Monoclonal epithelial markers panCK and EMA are used for IHC that can detect tumor cells and micrometasteses which are simply missed on routine H\&E. ${ }^{[5]}$

\section{Materials and Methods}

Total 36 patients from Jan 2001 to Jan 2005 having early breast cancers with distant metasteses free, underwent surgery at the hospital. All these patients had undergone modified radicle mastectomy along with axillary lymph node dissection (ALND) and were found to be $\mathrm{pNO}$ or $\mathrm{pN} 1$ on routine hematoxylin and eosin. All H\&E slides of tumor and lymph nodes were reviewed by pathologists. The relevant paraffin blocks with N0,N1 nodal status were retrieved from archives. Each block was serially sectioned.
Ten sections of $5 \mu \mathrm{m}$ thickness at $50 \mu \mathrm{m}$ interval were cut whenever possible. Out of these two sections were taken on poly-L- lysine coated microscope slides. These sections were subjected to IHC and rest were stained with $\mathrm{H} \& \mathrm{E}$.

The section numbering 5 and 10 were taken for immunostaining by pan CK and EMA for picking up micrometasteses. Both positive and negative controls were run with every batch of immunostaining.

\section{Results}

Any tumor cells visible on H\&E stained sections were distinguished between macro and micrometasteses. A total number of 463 lymph nodes from 36 cases of carcinoma breast were studied. New serial sections (SMS) cut from the 435 lymph nodes of the breast revealed $16(3.67 \%)$ additional positive lymph nodes from 12 cases. Out of 16 positive lymph nodes 4 were macrometasteses and 12 were micrometasteses, which were confirmed on IHC. Micrometastses in regional and axillary lymph nodes appeared mainly as single cells and as small clusters of 2-4 carcinoma cells (Figure 2,3), which were unrecognizable by conventional H\&E staining (Fig 2,3). Immunostining with cytokeratin $(\mathrm{CK})$ antibody revealed micrometasteses in 40 of $435(9.19 \%)$ lymph nodes and by epithelial membrane antigen(EMA) antibody 33 of 435 (7.59\%). Out of 40 micrometasteses 8 were positive for isolated tumor cells. The trend of increasing yield of lymph nodes by using serial multiple sections and immunohistochemistry is depicted in charts and figures as (Chart-1 table-1) and (figures- 1,2,3).

Equal number of cases were found in category of age groups less than 50 years and more than 50 years (chart 2 ). In case of breast carcinoma, the commonest site is upper outer quadrant of left followed by upper outer quadrant of right (chart - 3).

We used CK and EMA for carcinoma breast and found $\mathrm{CK}$ is the superior marker in picking up more number of micrometasteses (chart- 4)

Results of these experiments were interpreted statistically. We calculated the statistical inferences by using tests of significance. These tests are mathematical methods by which the probability (p) or relative frequency of an observed difference, occurring by chance is found. For this we used $\mathrm{Z}$ test. This test express the difference observed in terms of standard error (SE) which is a measure of variation in sample estimates that occur by chance.

We calculated the $Z$ value and corresponding $p$ value between two methods as H\&E versus SMS, H\&E versus IHC and SMS versus IHC. (Table 2). The tests revealed 
that serial multiple sections and immunohistochemistry are definitely the superior methods in detection of more number of micrometasteses in carcinoma breasts.
Out of 36 cases 6 cases showed change in staging and 3 out of 6 cases were upstaged from early to advanced stage carcinoma (chart-5)

Table 1:

\begin{tabular}{|l|l|l|}
\hline & YIELD OF POSITIVE LYMPH NODES & \\
\hline METHOD & NO/TOTAL & $\%$ \\
\hline HE & $28 / 463$ & 6.04 \\
\hline SMS+HE & $44 / 463$ & 9.5 \\
\hline IHC+HE & $68 / 463$ & 14.68 \\
\hline
\end{tabular}

Table 2:

\begin{tabular}{|c|c|c|c|c|}
\hline \multicolumn{5}{|c|}{ CARCINOMA BREAST } \\
\hline & $M+V E$ & M -VE & Z- VALUE & $p$ - VALUE \\
\hline $\mathrm{HE}$ & 28 & 435 & 1.97 & $<0.05$ (S) \\
\hline SMS & 44 & 419 & & \\
\hline $\mathrm{HE}$ & 28 & 435 & 4.38 & $<0.01(\mathrm{~S})$ \\
\hline $\mathrm{IHC}$ & 68 & 395 & & \\
\hline SMS & 44 & 419 & 2.43 & $<0.02(\mathrm{~S})$ \\
\hline $\mathrm{IHC}$ & 68 & 395 & & \\
\hline
\end{tabular}

Table 3: Micrometasteses and detection rate in carcinoma breast.

\begin{tabular}{|l|l|l|l|}
\hline S.No & Authers & Method & Positivity \\
\hline 1 & Saphir \& Amronin 1948(17) & H\&E & $33 \%$ \\
\hline 2 & Pickren 1961(8) & H\&E & $22 \%$ \\
\hline 3 & Fisher et al 1978 (20) & H\&E & $24 \%$ \\
\hline 4 & Rosen et al 1981 (10) & H\&E & $32 \%$ \\
\hline 5 & Busollatti et al 1986 (23) & IHC & $20 \%$ \\
\hline 6 & Wells et al 1987 (11) & IHC & $15.5 \%$ \\
\hline 7 & Ludwig Breast Study Group (22) & Serial H\&E & $9 \%$ \\
\hline 8 & de Mascarel et al 1992(12) & Serial H\&E & $14 \%$ \\
\hline 9 & Hainsworth et al 1993 (14) & IHC & $12 \%$ \\
\hline 10 & Elson et al 1993 (15) & IHC & $20.6 \%$ \\
\hline 11 & Clare et al 1997 (13) & IHC & $12.8 \%$ \\
\hline 12 & Millis et al 2002 (24) & IHC & $12.5 \%$ \\
\hline 13 & Present study & H\&E, IHC & $14.68 \%$ \\
\hline
\end{tabular}

H\&E - Hematoxylene and Eosin; IHC - Immunohistochemistry ; SMS - Serial Multiple Sections. 


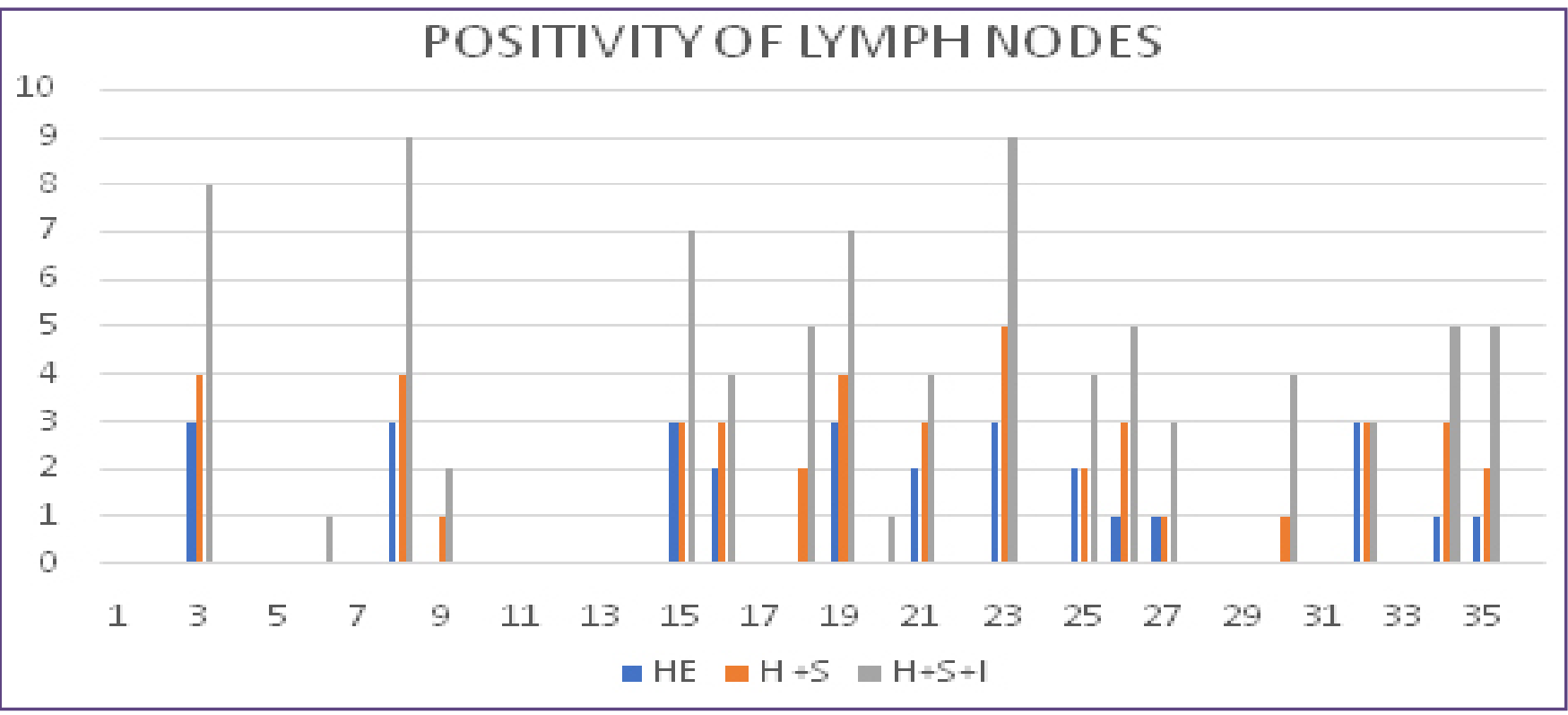

Chart -1

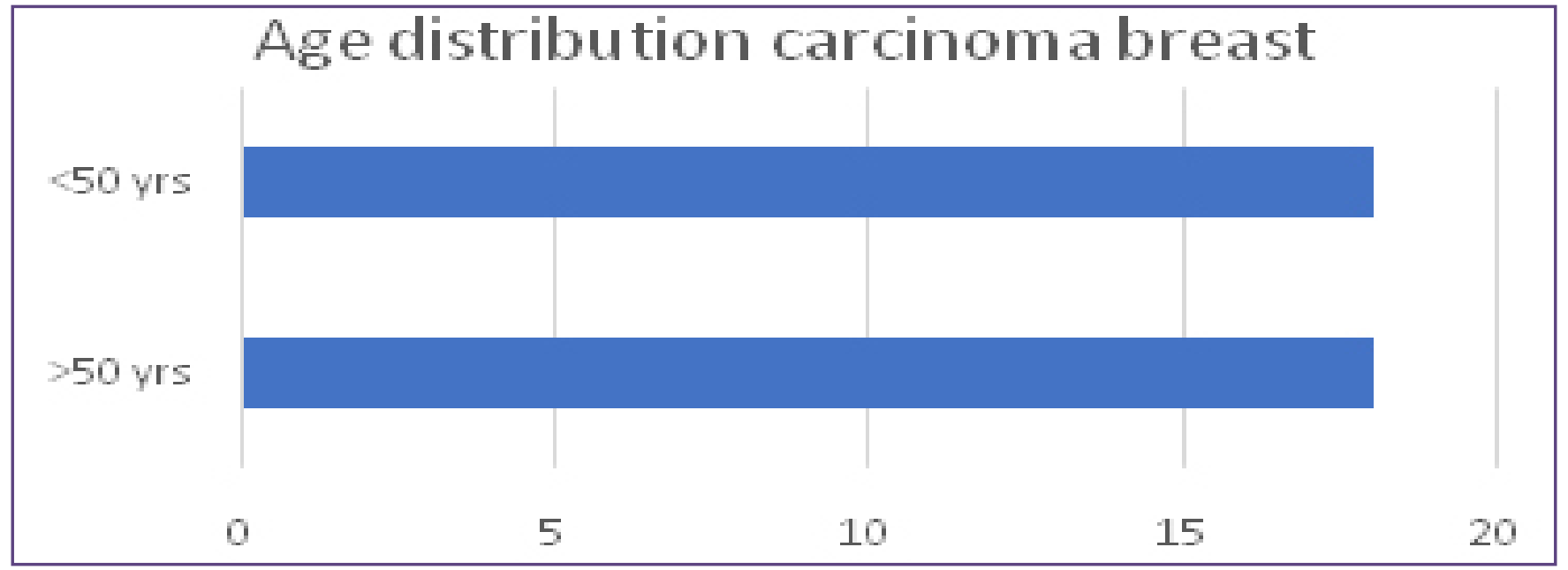

Chart -2

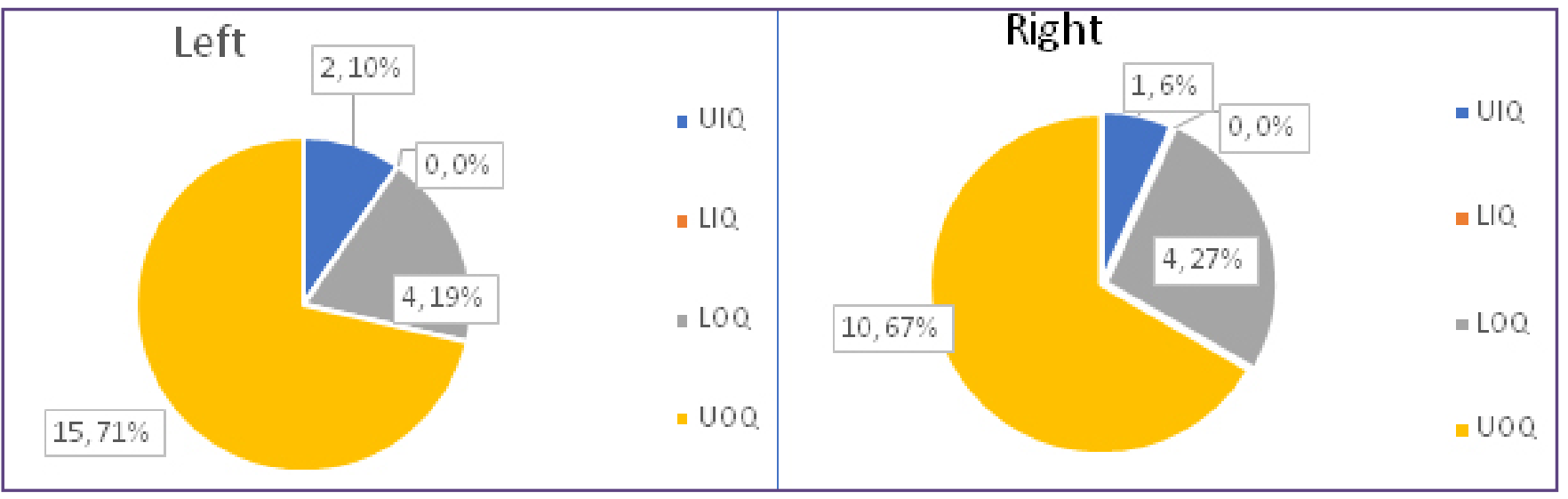

Chart -3 


\section{POSITIVITY FOR MARKERS}

$$
\begin{array}{r}
45 \\
40 \\
35 \\
30 \\
25 \\
20 \\
15 \\
10 \\
5 \\
0
\end{array}
$$

$\mathrm{CK}$

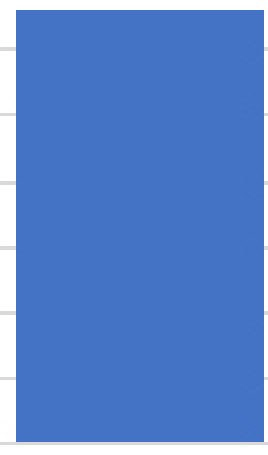

EMA

Chart -4

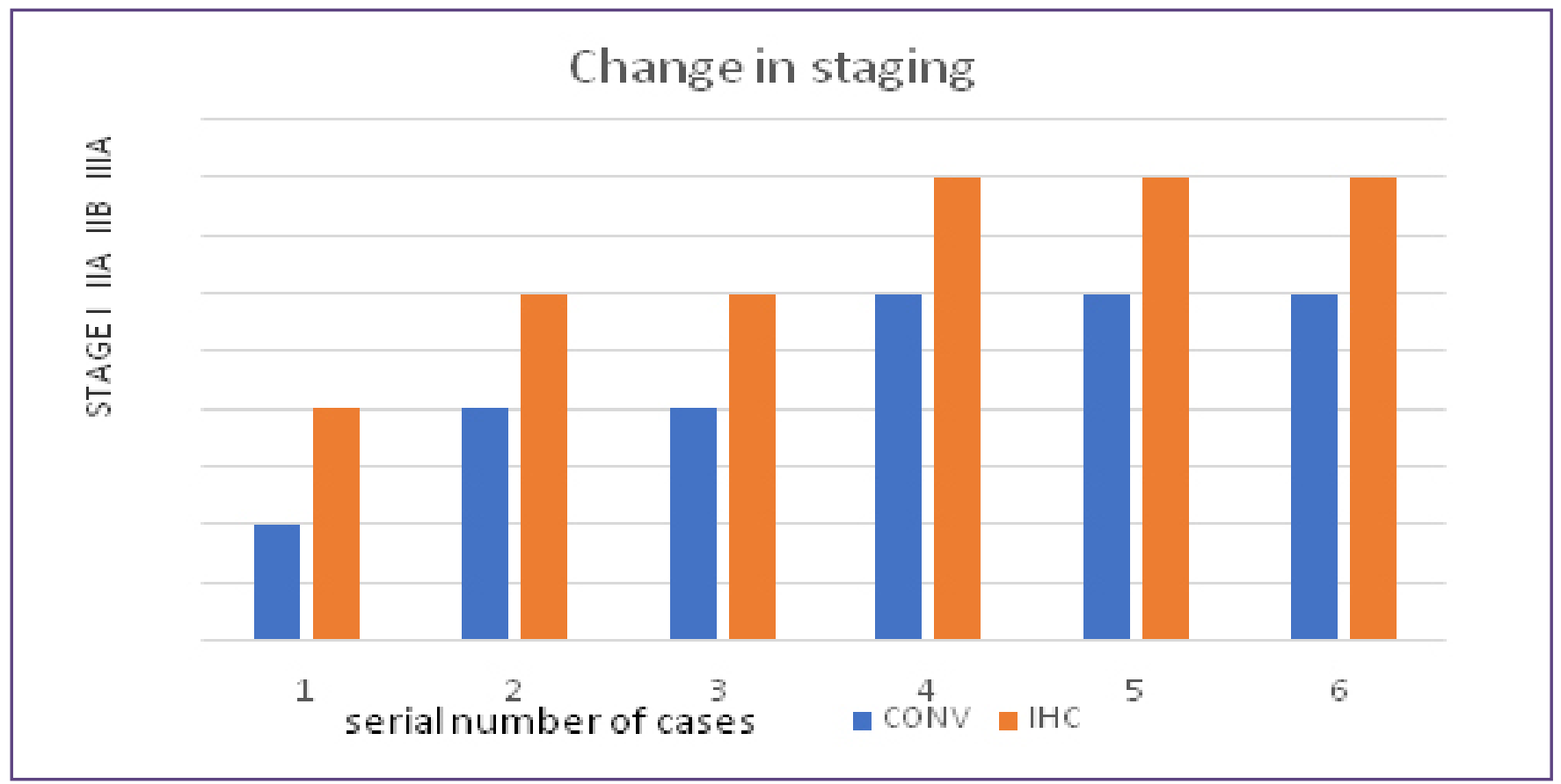

Chart -4 


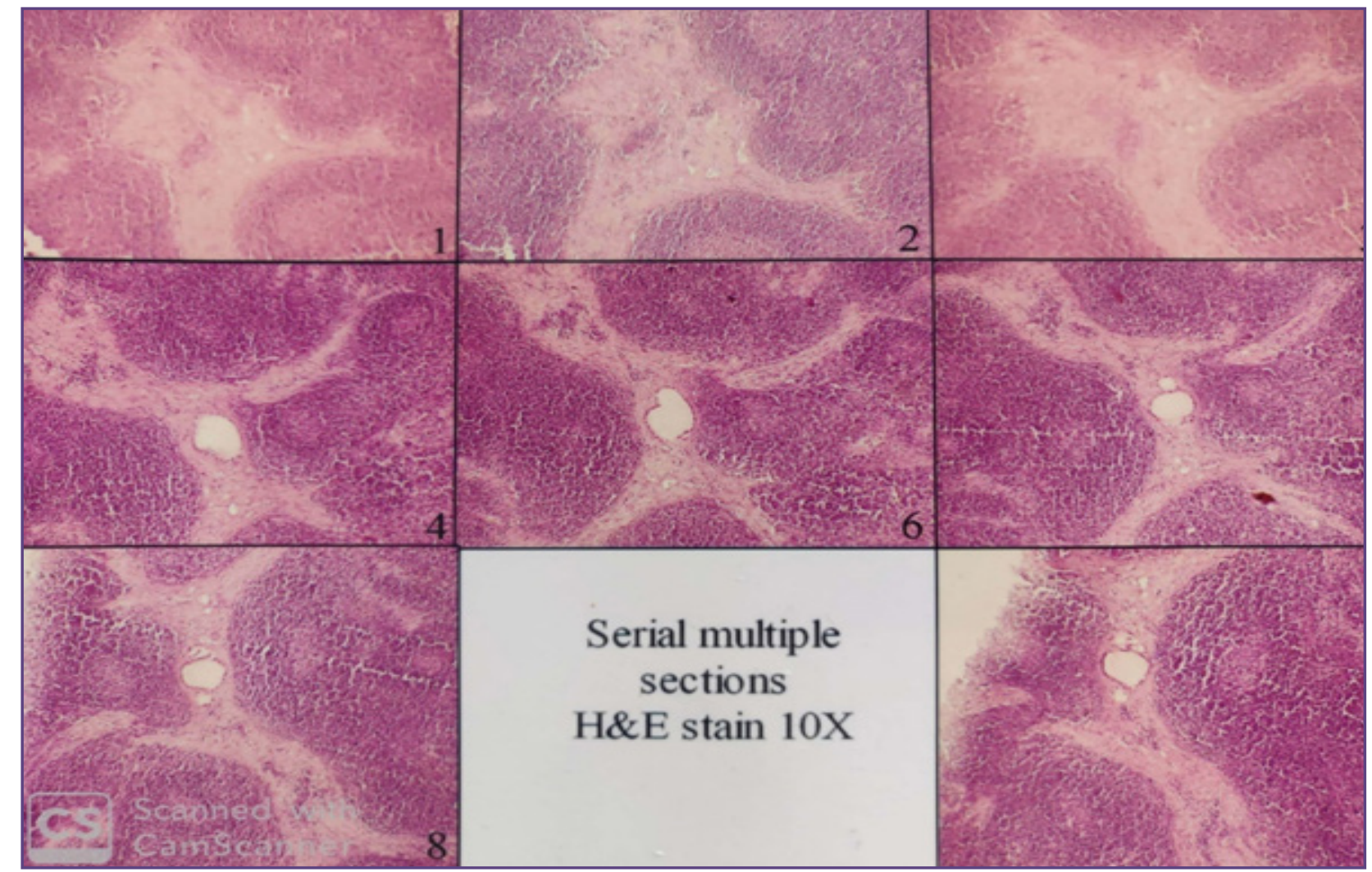

Fig. 1: SMS of a lymph node from ca breast reveals emerging micrometastesis in later sections.

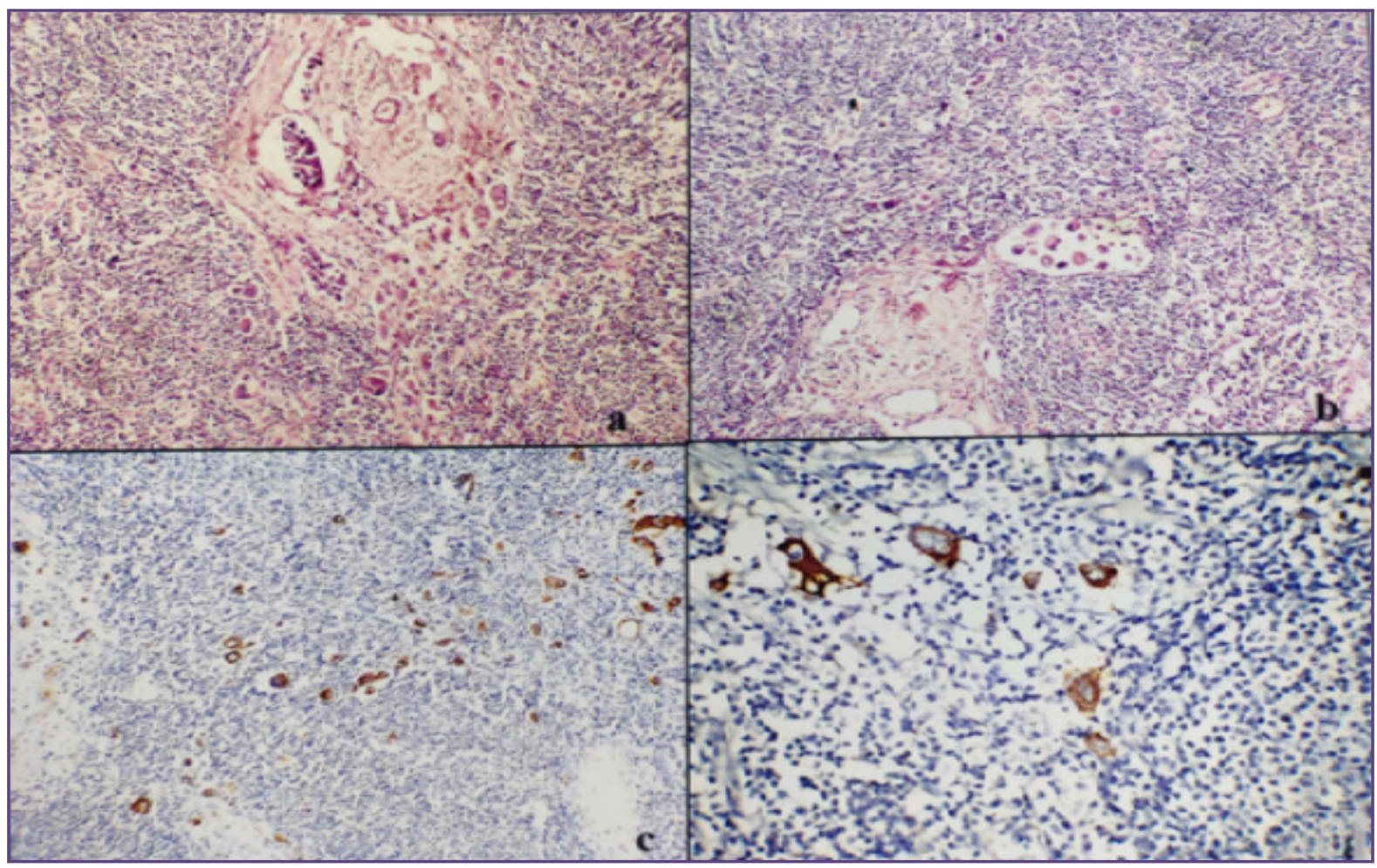

Fig. 2: A micrometastesis in breast carcinoma,H\&E; b- ITC in the lymphatics ; $\mathrm{c}$ d - ITC- Diffuse cytoplasmic CK positivity 


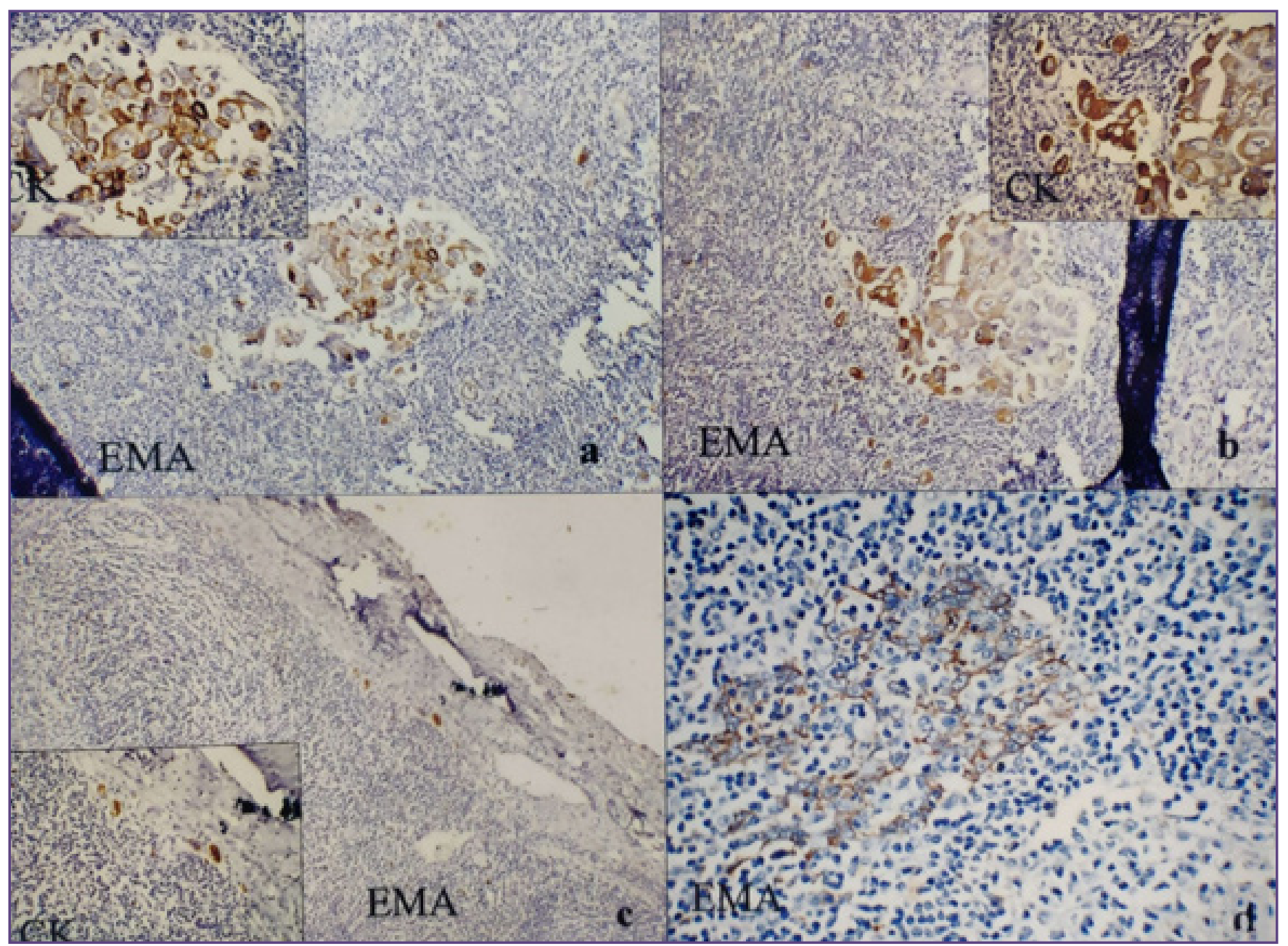

Fig. 3: Immunostaining with CK and EMA for carcinoma breast in a lymph node.

\section{Discussion-}

In the present study, an analysis of the significance of serial multiple sections and immunohistochemistry over conventional $\mathrm{H}$ and $\mathrm{E}$ stains in detection of lymph nodes metasteses and the subsequent change in staging has been carried out.

The mean age of our patients was 51 years which is in contrast with Indian average of 42 years. In our hospital, systematic lymphadenectomy is performed in operable cancer cases. The resected nodes were dissected, labelled and all nodes were examined pathologically.

The behavior and prognosis of cancers are generally stage dependent. Staging of tumor requires proper assessment of draining lymph nodal status in addition to primary lesion and the presence or absence of metasteses. Pre and postoperatively pathological lymph node status provides the most accurate and critical information, so that relevant clinical management decisions can be taken with regards to further surgeries and adjuvant therapies. Micrometastases or isolated tumor cells in the regional lymph nodes were associated with an absolute reduction in the 5-year rate of disease-free survival of nearly 10 percentage points. ${ }^{[4]}$ Patients with node positive breast cancer have a high risk of recurrence and cancer related death and should receive adjuvant therapy. In one ASCO study in 2009 analyzed 1000 cases ITC/micromets and found that the chance of recurrence in patients with micromets is 5 times more than the node-negative patients if there is no further treatment given, in the form of ALND or radiotherapy. ${ }^{[5]}$

The size of primary tumor and the axillary node status are the two most important prognostic indicators of breast carcinoma. ${ }^{[9]}$ Advantages of systemic therapy may be of benefit in both pre and postmenopausal lymph node negative patients. The traditional method of evaluating lymph nodes for metaseteses has been shown to be unreliable. ${ }^{[10,11,12,13,14,15,16,17]}$ Occult metastases are not observed during the initial routine histopathologic 
evaluation but become apparent at deeper levels of routine histologic sections or on IHC evaluation. ${ }^{[17]}$ To take a correct decision for the mode of therapy by surgeons, we need to identify true negative lymph nodes and lymph nodes with occult micrometastesis. ${ }^{[3,17]}$ Micrometastesis, is the tumor deposits based on size, with cutoff point ranging from 0.2-2.0 mm. ${ }^{[3,4,5,17]}$ Presence of increasing number of micrometasteses has been shown to be associated with poor prognosis. Added adjuvant chemotherapy is required after radical surgery.

The increased yield of serial sections with H\&E staining and IHC has been reported variously as ranging from $7-32 \% \quad[10,11,12,13,14,15,16,18,19,20,21,22]$ (Table-3). Some of the early studies failed to show any significance of occult metasteses, whereas more recent studies with larger population of patients, longer follow up and use of IHC have shown these differences to be significant. ${ }^{[4,5,10,12,14,21]}$ In our study it has been seen that an increased number of micrometaseteses could be identified from $6.04 \%$ with routine $\mathrm{H} \& \mathrm{E}$ section to $9.5 \%$ with serial multiple sections (SMS). The yield increased further to $14.68 \%$ with the use of immunohistochemistry. An increasing trend of positivity was detected and CK was found to be better marker than EMA. ${ }^{[3,5]}$ In our study, serial multiple sections and immunohistochemistry has significantly increased the yield of metastatic lymph node which led to subsequent change in staging of 3 cases.

Impact of serial sections found in this study with sections of $5 \mu \mathrm{m}$ thickness at $50 \mu \mathrm{m}$ interval is that we had a yield of $(9.5 \%)$ lymph nodes over $(6.04 \%)$ on routine $(\mathrm{P}-<0.05)$.

Impact of immunohistochemistry is that a number of studies have shown increased yield (9-30\%) of metastatic lymph nodes by using monoclonal antibodies for IHC. ${ }^{[5]}$ In this study too we had a yield of $14.68 \%$ positivity on IHC compared to $6.04 \%$ on routine H\&E staining of breast cases ( $\mathrm{p}-<0.01)$. Isolated tumor cells have been grouped in the $\mathrm{pN} 0$ stage in case of breast cancers.

$\mathrm{H} \& \mathrm{E}$ - Hematoxylene and Eosin ; IHC Immunohistochemistry ; SMS - Serial Multiple Sections

\section{Conclusion -}

Significant number of micrometasteses are missed by routine processing of lymph nodes and $H \& E$ staining. Serial multiple sections of lymph nodes increase the chance of detecting increased micrometasteses though but is time consuming and not practical as a routine laboratory procedure. However Immunohistochemistry is rapid,convenient and detects increased micrometasteses in lymph nodes hence can be considered as routine procedure in node negative and high risk patients particularly.
We also observed Cytokeratin as superior marker than EMA. Continuous efforts to define "clinically relevant micrometasteses" should persist.

\section{References}

1. Siegel R, DeSantis C, Virgo K, Stein K, Mariotto A, Smith $\mathrm{T}$, et al. Cancer treatment and survivorship statistics. CA Cancer J Clin. 2012;62(4):220-41.

2. Abigail S Caudle, Julie A Cupp, Henry M Kuerer. Management of Axillary Disease. Surg Oncol Clin N Am. 2014 ;23(3):473-86.

3. Yadav R, Singh S, N Marwah, K Kataria, G Aggarwal, R Sen. Immunohistochemical detection of axillary lymph node micrometastases in breast cancer patients: Increasing the accuracy of detection and decreasing labor intensive serial sectioning. Indian J Cancer 2014; 51(3):267-271

4. Maaike de B, Carolien H.M. van Deurzen, George F. Borm, Paul. van Diest, Eddy M.M. Adang et al. Micrometastases or Isolated Tumor Cells and the Outcome of Breast Cancer. N Engl J Med 2009; 361:653-663

5. Indu RN, Anand JM, Vijaykumar DK. Detection of micrometastasis in axillary lymph nodes of breast carcinoma patients and its association with clinical outcome. Indian J Pathol Microbiol. 2018; 61 ( 3 ): 330-33

6. Donald L.W, David N.K, Edward A.M, Takamaru A, Brenda L. W, Seth P. H et al. Detection of Occult Sentinel Lymph Node Micrometastases by Immunohistochemistry in Breast Cancer An N S A B P Protocol B - 32 Quality Assurance Study. Cancer 2006;107:661-7.

7. Jeremy St. T. Retrieval, handling and assessment of lymph nodes in cancer resection specimens. Current Diagnostic Pathology 2006; 12,75-82.

8. Patricia T, Kurian J. J, and Edward Y. Issues Related to Sentinel Lymph Node Assessment in the Management of Breast Cancer-What Are Relevant in Pathology Reports? Pathology Research International 2011; 7

9. Rampaul R, Miremadi A, Pinder S, et al. Pathological validation and significance of micrometastesis in sentinel nodes in primary breast cancer. Breast cancer Res 2001;3:113-116

10. Rosen PP, Saigo P, Braum DW Jr, et al. axillary micro and macrometasteses in breast cancers; prognostic significance of tumor size. Ann Surg.1981;194(5):585-91.

11. Wells CA, Heryet A, Brochier J, Gatter KC, Mason DY. The immunohistochemical detection of micrometasteses in breast cancer. Br J Cancer 1987;55(3):392-8.

12. de Mascarel I, Bonichon F, Coindre JM, Trojani M. Prognostic significance of breast cancer axillary lymph node micrometasteses assessed by two special techniques: reevaluation with longer follow up. $\mathrm{Br} \mathrm{J}$ Cancer. 1992;66(3);523-722.

13. S E Clare, S F Sener W. Wilkens R, et al. Prognostic significance of occult lymph node metasteses in nodenegative breast cancer. Ann Surg Oncol 1997;4:447-51. 
14. Hainsworth PJ, Tjandra J, Stillwell RG,et al. Detection and significance of occult metasteses in node negative breast cancer. Br J Surgery 1993;80:459-63

15. Elson CE, Kulfe D, Johnson WW. Immunohistochemical detection and significance of lymph node micrometasteses in breast carcinoma. Anal Quani Cytol Histol 1993;15:171-8.

16. J. Reed, M. Rosman, K.M. Verbanac, et al. Prognostic implications of isolated tumor cells and micrometastases in sentinel nodes of patients with invasive breast cancer: 10year analysis of patients enrolled in the prospective. J Am Coll Surg 2009;208:333-340

17. Aysegul A. Sahin, Merih Guray, and Kelly K. Hunt. Identification and Biologic Significance of Micrometastases in Axillary Lymph Nodes in Patients With Invasive Breast Cancer. Arch Pathol Lab Med 2009;133:(6) 869-878.
18. A.E. Giuliano, K.K. Hunt, K.V. Ballman, et al. Axillary dissection vs no axillary dissection in women with invasive breast cancer and sentinel node metastasis: A randomized clinical trial JAMA;2011: 569-575.

19. Neville AM, Price K, Gelber RD, Goldhirsch A. Axillary node micrometastases and breast cancer. Lancet 1991;337(8749): 1110.

20. Bussolatti G, Gugliotta P, Morra J, Pietribiasi F, Berardengo E. The immunohistochemical detection of lymph node metasteses from infilterating lobular carcinoma of the breast. Br J Cancer 1986; 54(4):631-6.

21. Millis RR, Springall R, Lee AHS, et al. Occult axillary lymph node metasteses are of no prognostic significance in breast cancer. Br J Cancer 2002;86:396-401

22. V. Galimberti, B.F. Cole, S. Zurrida, et al. Axillary dissection versus no axillary dissection in patients with sentinel-node micrometastases Lancet Oncol;14 (2013): 297-305.

*Corresponding author:

Dr. Sunita Sharma, C/O SurgCapt CS Mohanty, P-408/1, PerieraEnclave,MinnieBay,Port Blair, (A \& N islands) Pin -744103, India

Phone: +91 9757167347

Email: 1tsunita@hotmail.com

Date of Submission : 01/12/2019

Financial or other Competing Interests: None. 